\title{
Effect of Continuity Rate on Multistage Logistic Network Optimization under Disruption Risk
}

\author{
Muhammad Rusman* \\ Department of Industrial Engineering, Hasanuddin University, Makassar, Indonesia \\ Yoshiaki Shimizu \\ Department of Mechanical Engineering, Toyohashi University of Technology, Toyohashi, Japan
}

(Received: January 31, 2013 / Revised: May 29, 2013 / Accepted: May 29, 2013)

\begin{abstract}
Modern companies have been facing devastating impacts from unexpected events such as demand uncertainties, natural disasters, and terrorist attacks due to the increasing global supply chain complexity. This paper proposes a multi stage logistic network model under disruption risk. To formulate the problem practically, we consider the effect of continuity rate, which is defined as a percentage of ability of the facility to provide backup allocation to customers in the abnormal situation and affect the investments and operational costs. Then we vary the fixed charge for opening facilities and the operational cost according to the continuity rate. The operational level of the company decreases below the normal condition when disruption occurs. The backup source after the disrup-tion is recovered not only as soon as possible, but also as much as possible. This is a concept of the business continuity plan to reduce the recovery time objective such a continuity rate will affect the investments and op-erational costs. Through numerical experiments, we have shown the proposed idea is capable of designing a resilient logistic network available for business continuity management/plan.
\end{abstract}

Keywords: Disruption Risk, Mixed-Integer Programming, Resilient Logistic Network, Continuity Rate

* Corresponding Author, E-mail: rusman@tiunhas.net

\section{INTRODUCTION}

Dealing with supply chain disruptions has been a challenging issue for companies under recent globalization. They are unplanned and unanticipated events that disrupt the normal flow of products and materials within a supply chain. The disruption at one member of supply chain can result in a significant impact on the entire chain. Supply chains are subject to potential external sources of disruption such as natural disasters and terrorist attacks.

If we look back at the natural disasters in the recent decade: the latest earthquake and tsunami in Japan in March 2011, devastating floods in Thailand, and extreme winter in Europe in early 2012, supply chain activities in companies have been put at the edge of high risk due to frequent natural events that bring catastrophic impact to companies. Issues mentioned above can bring devastating impacts on the company's operations and in particular on its supply chain and logistic activity. Moreover, such disruptions in the supply chain are not only increasing in frequency, but the severity of their impact is escalating in terms of costs and losses. They can bring a company to a partial or complete halt.

We can anticipate the disruption by considering preventive action to ensure that the supply chain is not adversely affected. If the supply chain takes preventive action against the disruption, such action is viewed as mitigation planning. Under such a mitigation plan, the supply chain must build a robust system that will minimize the impact of the disruption in the future. One such mitigation mechanism would be to have backup 
facilities that may provide supplies if the primary facility would be disrupted. Schmitt (2011) recommended that one of the best protections can be achieved through backup capabilities that will protect the supply chain until the disruption's end and prevent long or permanent interruptions to customer.

This paper is focused on studying a multistage logistic network where the facility can be disrupted partially and still be able to serve below its capacity. Therefore, we consider applying a continuity rate for facilities. This consideration is not taken into account in the previous studies (Rusman and Shimizu, 2011, 2012; Shimi$\mathrm{zu}$ and Rusman, 2012). Consequently, the aim of the present study is to compare the properties of the previous model and the proposed model.

The remainder of the paper is organized as follows. Section 2 briefly surveys the relevant literature. Section 3 describes the model formulation of the problem. Section 4 presents numerical experiments and results. Section 5 concludes the paper and presents the future work perspectives.

\section{LITERATURE REVIEW}

Disruption risk management is one of the emerging topics in supply chain management in the past decade. Both academics and industrialists try to identify ways to manage the disruption risk and minimize the negative impact of supply chain interruptions (Tang, 2006). A disruption risk can be defined as the major disruptions caused by natural and man-made disasters such as floods, hurricanes, earthquakes, and terrorist attacks or economic crises such as currency fluctuation or employee strikes (Tang, 2006).

There are some previous studies on supply chain considering disruption risk. Snyder and Daskin (2005) have introduced several models based on traditional facility location problems, in which some facilities might fail with a given probability. They assumed that in normal circumstances, customers are assigned to primary facilities and other facilities will serve the customers if the primary facility fails. Yu et al. (2009) studied the impact evaluation on sourcing method in a two stage supply chain in the presence of disruptions risk.

Tomlin (2006) investigated the impact of considering unreliable facilities for the facility location problems. The assumption of Snyder and Daskin (2005) that facility may disrupt with a certain probability is relaxed by Berman et al. (2007), Lim et al. (2009), and Cui et al. (2010). Lim et al. (2009) studied on the facility reliability problem (FRP), which is extended from the uncapacitated facility location problem (UFLP). They studied the FRP from the aspect of how to design a reliable supply chain network in the presence of random facility disruption. Reliable network design also is considered as a strategic supply chain management model that can per-form well under normal and abnormal condition (Peng et al., 2011).

Chopra and Sodhi (2004) and Kleindorfer and Saad
(2005) studied the risk management perspective on supply chain disruption. These studies are concerned with two echelon logistic problems and solved only small size problems. Considering the risk associated with demand fluctuation, Shimizu et al. (2006) applied a flexibility analysis for a three echelon logistic problem. A scenario-based approach is taken to give a solution procedure by recourse model (Shimizu et al., 2011).

As the body of the literature about multistage logistic network design shows, mixed-integer programming (MIP) models are the common models used in this area. These models range from uncomplicated uncapacitated facility location models to complex capacitated multistage or multi-commodity models. The common objective of these models is to determine the least cost system design. The cost system usually involved tradeoffs among fixed opening costs of facilities and operational cost such as transportation costs handling cost and shipping cost. Melo et al. (2009) and Klibi et al. (2010) presented comprehensive reviews on supply chain network problems to support a variety of future research directions.

Risk can occur in every level of a supply chain, and it is recognized as a fundamental link in operating the overall activities and providing value to both firms and customers. Supply chain disruption risk can be defined as the unpredictable or uncertainty of events that can interrupt the overall supply chain or event with a probability that may happen with negative consequences to the supply chain (Tang and Nurmaya Musa, 2011). Uncertainty in demand such as demand fluctuation is one of the risk sources in supply chain. A method that can be applied to solve this problem is the flexibility analysis, which can be applied in multi-echelon logistic network (Shimizu et al., 2008).

In the previous literatures, most of the models assume that a disrupted facility cannot fulfill a part of their assigned demand with available resources. This assumption is not applicable in the real word situations since each facility might fail partially and still able to serve below the expected capacity. Therefore, we consider applying the continuity rate on the facility, where unreliable facility still can provide demand and also reliable facility decrease its backup ability in an abnormal situation. In this paper, we propose a multi stage logistic network design model, which is formulated as MIP models by considering the effect of the continuity rate under disruption risk.

\section{MODEL FORMULATION}

Throughout this paper, we are concerned with three echelon logistic problems, consisting of distribution centers (DCs), relay stations (RSs), and customers (REs). The location decisions are made in the RS level. We proposed two kinds of RS: reliable RS (RRS) and unreliable RS (URS). URS is subject to failures. Failure of the RS means that RS is no longer available to serve customers. When RS fails, the firm has to find alternate 
sources of supply to provide service to customers. RRS is the hardened facility, which has additional capacity and or external alternative sourcing strategy.

We consider three allocation models, namely, multimulti allocation model (MMA), multi-single allocation model (MSA), and single-single allocation model (SSA). In MMA model, relay station (RSj) and customer $k$ can receive product from multiple sources. In MSA model, only relay station $(\mathrm{RS} j)$ can receive product from multiple sources while customer $k$ only receives the product from a single source. In SSA model, both relay station $(\mathrm{RS} j)$ and customer $k$ only receive the product from a single source. Figure 1 shows the differences of configuration among them.

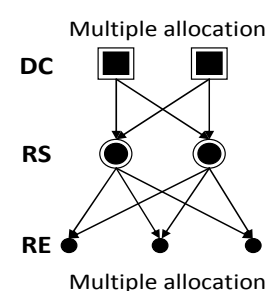

(a)

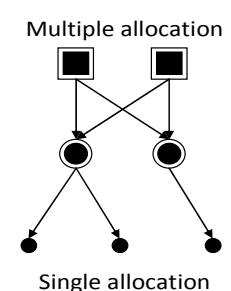

(b)

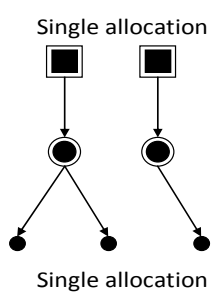

(c)
Figure 1. Allocation model: (a) multi-multi allocation model, (b) multi-single allocation model, and (c) single-single allocation model. DC: distribution center, RS: relay station, RE: customer.

Figure 2 illustrates the three echelon logistic network where RS is potentially being disrupted. Thereat, two DCs distribute products to three RSs, which consist of two RRS and one URS. If the demand of the customer (RE $i$ ) is satisfied by RRS, then only a single assignment is sufficient. On the other hand, if the customer $(\mathrm{RE} j)$ is assigned to URS, backup assignment is required besides the primary assignment. This means that when the disruption occurs at URS, demand of the customer will be distributed from RRS which is assigned as a backup for the customer (RE).

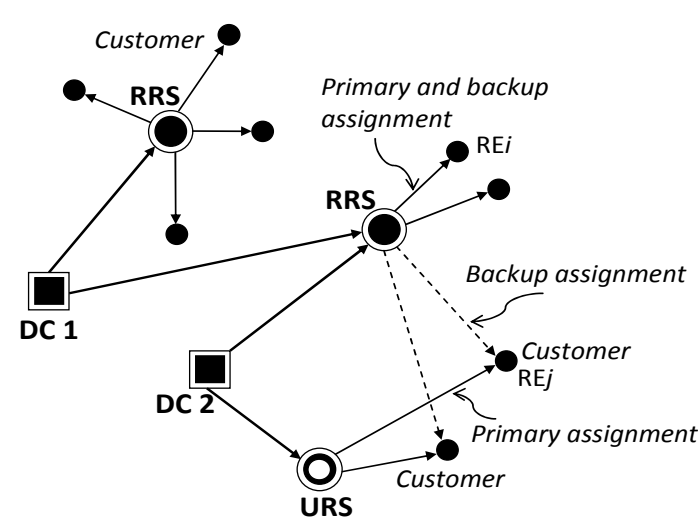

Figure 2. Illustration of the logistic network model under disruption risk. DC: distribution center, RRS: reliable relay station, URS: unreliable relay station, REj: customer.

\section{$3.1 w / o$ and $w$-model}

In this paper, we introduce two kinds of model comparison, namely, without model or $w / o$-model and with model or $w$-model. The $w / o$-model is a previous assumeptions model without considering the continuity rates on multistage logistic network design considering disruption risk (Rusman and Shimizu, 2011, 2012). In this model, we assume that the URS is completely halted when disruption occurs and the backup assignment is only from RRS.

The $w$-model is a new model that takes into account the continuity rates. We assumed in an abnormal situation $w$-model is more robust and flexible where backup allocation can be supplied from both facilities, RRS and URS. Backup capacities are dependent on the value of continuity rate of the facilities. In this situation, URS still can supply the product to customer as a backup assignment with a certain amount depending on the continuity rate $\left(r^{U}\right)$. On the other hand, backup capacity of RRS depends on the continuity rate of RRS $\left(r^{R}\right)$. The continuity rate value $\left(r^{U}, r^{R}\right)$ is related to the investment cost for opening facility. When the $r^{U}$ is high, then the investment cost of the facility becomes expensive, but the consequence of this cost is that the backup capacity of URS becomes higher in an abnormal situation. This consideration leads to amending the network to be more robust and flexible in a disruption situation. This assumption is applicable in the real world applications.

In the $w / o$-model, once the network is disrupted, the model intensifies to open more reliable RSs rather than unreliable ones. On the contrary, the $w$-model will optimize the backup capacity of URS before considering additional backup supply from other RRS. We illustrate the robustness and flexibility of the $w$-model compared to the $w / o$-model in Figure 3. The main difference between these models is when disruption occurs, the URS still can supply product to customer as backup assignment.

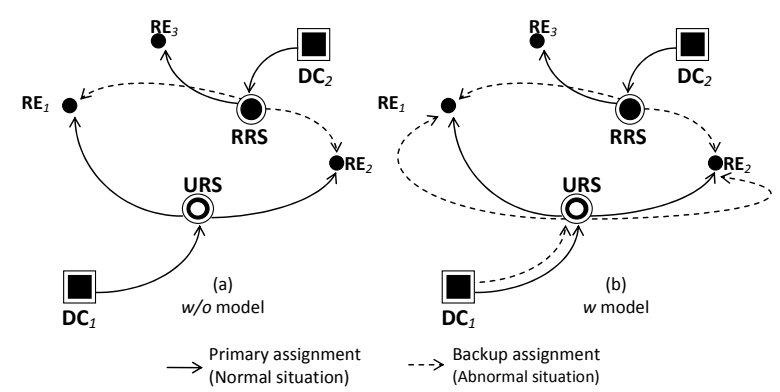

Figure 3. The difference configuration for $w / o$ and $w$ model. DC: distribution center, RRS: reliable relay station, URS: unreliable relay station, RE: customer.

Figure 4 depicted the comparison of the representative scheme of the continuity rate of the $w / o$ - and $w$ model. After the disruption, the operational level decreases somewhat below the normal condition even for 
the RRS while URS can keep it at a certain level. Thus, the continuity rate is viewed as the operational level during the required time objective after the disruption.

When disruption occurs in the facility, the operational level of supply chain activity will decrease below the normal condition. The backup facility will cover the demand of customers in the abnormal condition until the system achieves the recovery state as a normal condition. How fast the system can recover from abnormal condition depends on the continuity rate of facilities and operational supply chain activities. This is the basic concept of the $\mathrm{BCP} / \mathrm{M}$, whose purpose is to reduce recovery time objective (RTO) as short as possible. In other words, it must involve a preventive and remediable plan against emergency for management and/or decision making to maintain the business continuously.

The concept of continuity rate can be applied to increase the supply flexibility by considering multiple sourcing strategy for mitigating disruption. Under normal circumstances, an organization can improve the capability to manage supply, and after a major disruption, is able to adjust order quantity quickly.

\subsection{Continuity Rate}

Here we introduce the continuity rate of reliable and unreliable $\mathrm{RS}\left(r^{R}, r^{U}\right)$, continuity rate of shipping $\left(r_{p}\right)$, continuity rate of handling $\left(r_{h}\right)$ and the decreasing rate in demand $\left(r_{d}\right)$ on the extended model. The value of the continuity rate is denoted in general by $r(0<r \leq 1)$ for $r=\left\{r^{R}, r^{U}, r_{p}, r_{h}, r_{d}\right\}$.

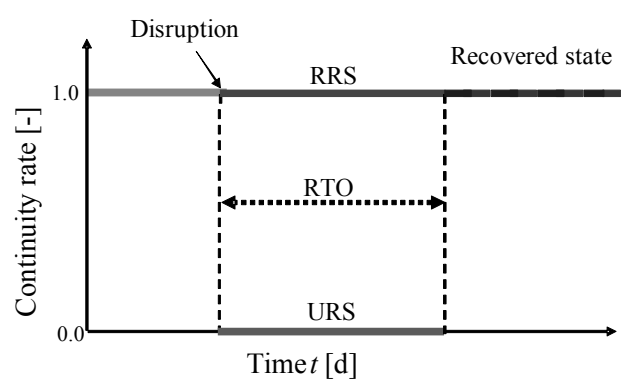

(a)

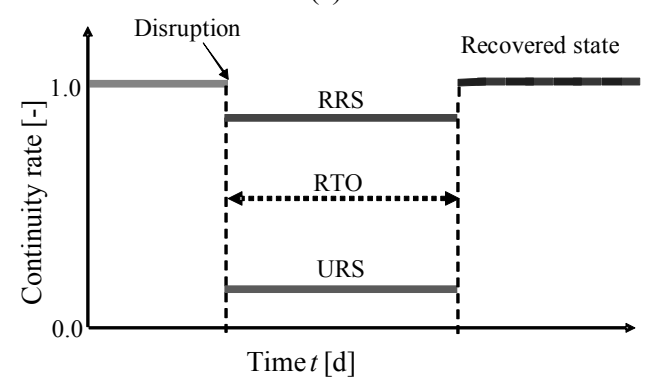

(b)

Figure 4. Comparison of continuity rates: (a) in case of conventional $w / o$-model, (b) in case of present $w$ model. RRS: reliable relay station, URS: unreliable relay station, RTO: recovery time objective.

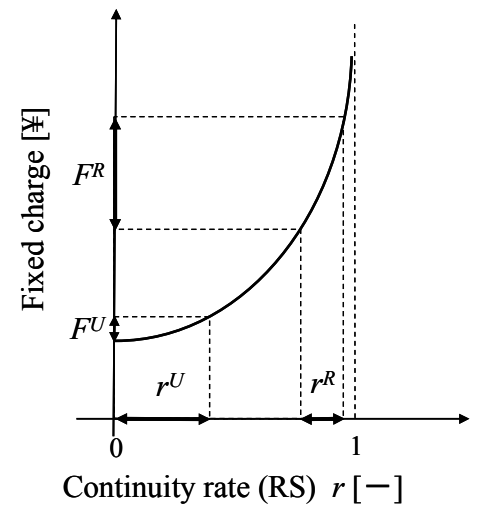

Figure 5. Scheme of fixed charge against continuity rate. RS: relay station.

The fixed cost for opening RS follows the exponential function as shown in Figure 5. Generally speaking, building RS with a higher continuity rate needs a higher investment cost or fixed charge and ultimately an infinite cost for the perfect reliability. This means that we can describe the fixed charge as an exponential function of the continuity rates. It is described as $r^{U}$ and $r^{R}$ for URS $\left(F^{U}\right)$ and RRS $\left(F^{R}\right)$, respectively. The fixed cost for opening RS can be obtained by following the equation.

$$
F=\alpha \exp (\beta r)+\gamma, \text { for } F=\left\{F^{U}, F^{R}\right\} \text { and } r=\left\{r^{U}, r^{R}\right\}
$$

On the other hand, the relation between the operational costs such as shipping cost at DC or handling cost at $\mathrm{RS}$, and the continuity rate has the opposite trend to such fixed charge. We describe the continuity rate of shipping and handling tasks as $r_{p}$, and $r_{h}$, respectively. These operational costs also change with the continuity rate between upper and lower bounds. This change can be described by either linear or nonlinear function as depicted in Figure 6. We consider the primary shipping cost $C^{P}$ is equal to $C_{0}$ as the lower value of the shipping cost and $C_{1}$ as the upper value. The backup shipping $\operatorname{cost}\left(C^{B}\right)$ can be obtained by following the equation.

$$
C^{B}=r_{p} C_{0}+\left(1-r_{P}\right) C_{1}
$$

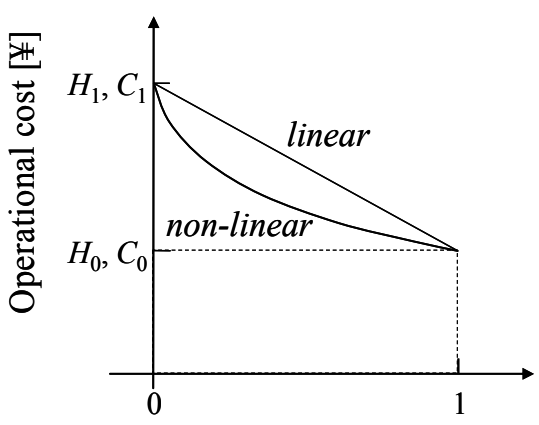

Continuity rate, $r_{p}(\mathrm{DC}), r_{h}(\mathrm{RS})[-]$

Figure 6. Operational cost against continuity rate. DC: distribution center, RS: relay station. 
Similarly, we apply the same relation to obtain backup handling cost. We consider that the primary handling cost $H^{P}$ is equal to $H_{0}$ as the lower value of the shipping cost and $H_{1}$ as the upper value. The backup handling cost $\left(H^{B}\right)$ can be obtained by following the equation.

$$
H^{B}=r_{p} H_{0}+\left(1-r_{P}\right) H_{1}
$$

Apparently, the primary cost of those (at normal state, $r_{p}=1$ ) corresponds to lower values. Generally, these continuity rates of operational costs are closely related with the fixed charge since the vulnerable RSs need higher cost and vice versa. For simplicity, we assume that it refers to the following linear model to represent this situation.

$$
r=f_{n}\left(r^{U}, r^{R}\right)=(1-w) r^{U}+w r^{R}, r \in\left\{r_{h}, r_{p}\right\}
$$

where $w$ denotes a weighting coefficient.

The following notations are used to describe the present mathematical models.

\section{Index sets}

$I \quad:$ set of DC

$J$ : set of RS

$K$ : set of RE

\section{Parameters}

$F_{j}^{U} \quad$ : Fixed cost for opening URS $j$

$F_{j}^{R} \quad$ : Fixed cost for opening RRS $j$

$C_{i}^{P} \quad$ : Shipping cost at DC $i$ as primary assignment

$C_{i}^{B} \quad$ : Shipping cost at DC $i$ as backup assignment

$H_{j}^{P} \quad$ : Handling cost at $\mathrm{RS} j$ as primary assignment

$H_{j}^{B} \quad$ : Handling cost at $\mathrm{RS} j$ as backup assignment

$T 1_{i j}^{P}$ : Transport cost from DC $i$ to $\mathrm{RS} j$ as primary assignment

$T 1_{i j}^{B} \quad$ : Transport cost from DC $i$ to RS $j$ as backup assignment.

$T 2_{j k}^{P}$ : Transport cost from $\mathrm{RS} j$ to customer $k$ as primary assignment

$T 2^{B}{ }_{j k}$ : Transport cost from RRS $j$ to customer $k$ as backup assignment

$U_{j} \quad$ : Capacity of RS $j$

$P U_{i}$ : Maximum supply ability of DC $i$

$P L_{i} \quad$ : Minimum supply ability of DC $i$

$d_{k} \quad$ : Demand of customer $k$

$q \quad$ : Probability of disruption $(0<q<1)$

$r^{R} \quad$ : Continuity rate of reliable facility

$r^{U} \quad$ : Continuity rate of unreliable facility

$r_{p} \quad$ : Continuity rate of production $\left(0<r_{p} \leq 1\right)$

$r_{h} \quad$ : Continuity rate of handling $\left(0<r_{h} \leq 1\right)$

$r_{d} \quad$ : Decrease rate in demand $\left(0<r_{d} \leq 1\right)$

\section{Decision variables}

$a_{i j}^{P} \quad$ : Shipped amount from DC $i$ to $\mathrm{RS} j$ as primary assignment.

$a_{i j}^{B} \quad$ : Shipped amount from DC $i$ to $\mathrm{RS} j$ as backup assignment.

$b_{j k}^{P} \quad$ : Shipped amount from RS $j$ to customer $k$ as primary assignment.

$b_{j k}^{B} \quad$ : Shipped amount from RS $j$ to customer $k$ as backup assignment.

$x_{j}^{U} \quad: 1$ if $\mathrm{RS} j$ is opened as unreliable one and 0 otherwise.

$x_{j}^{R}: 1$ if $\mathrm{RS} j$ is opened as reliable one and 0 otherwise.

$y_{j k}^{P} \quad: 1$ if $\mathrm{RS} j$ distributes customer $k$ as primary assignment and 0 otherwise.

$y_{j k}^{B} \quad: 1$ if $\mathrm{RS} j$ distributes customer $k$ as backup assignment and 0 otherwise.

$z_{i j}^{P} \quad: 1$ if DC $i$ distributes $\mathrm{RS} j$ as primary assignment and 0 otherwise.

$z_{i j}^{B} \quad: 1$ if DC $i$ distributes $\mathrm{RS} j$ as backup assignment and 0 otherwise.

\subsection{Multi-Multi Allocation Model}

The model for MMA is described as follows.

Minimize $\sum_{j \in J} F_{j}^{U} x_{j}^{U}+\sum_{j \in J} F_{j}^{R} x_{j}^{R}$

$$
\begin{aligned}
& +(1-q)\left(\sum_{i \in I} \sum_{j \in J}\left(C_{i}^{P}+T 1_{i j}^{P}\right) a_{i j}^{P}+\sum_{j \in J} \sum_{k \in K}\left(H_{j}^{P}+T 2_{j k}^{P}\right) b_{j k}^{P}\right) \\
& +(q) \quad\left(\sum_{i \in I} \sum_{j \in J}\left(C_{i}^{B}+T 1_{i j}^{B}\right) a_{i j}^{B}+\sum_{j \in J} \sum_{k \in K}\left(H_{j}^{B}+T 2_{j k}^{B}\right) b_{j k}^{B}\right)
\end{aligned}
$$

Subject to:

$$
\begin{aligned}
& x_{j}^{U}+x_{j}^{R} \leq 1 \quad \forall j \in J \\
& \sum_{j \in J} x_{j}^{R} \geq 1 \\
& \sum_{i \in I} a_{i j}^{P} \leq U_{j}\left(x_{j}^{U}+x_{j}^{R}\right) \quad \forall j \in J \\
& \sum_{i \in I} a_{i j}^{B} \leq U_{j}\left(r^{R} x_{j}^{R}+r^{U} x_{j}^{U}\right) \quad \forall j \in J \\
& \sum_{j \in J} a_{i j}^{P} \leq P U_{i} \quad \forall i \in I \\
& \sum_{j \in J} a_{i j}^{B} \leq r_{p} P U_{i}, \quad\left(0<r_{p} \leq 1\right) \quad \forall i \in I \\
& \sum_{j \in J} a_{i j}^{P} \geq P L_{i} \quad \forall i \in I \\
& \sum_{j \in J} a_{i j}^{B} \geq P L_{i} \quad \forall i \in I \\
& \sum_{i \in I} a_{i j}^{P}-\sum_{k \in K} b_{j k}^{P}=0 \quad \forall j \in J \\
& \sum_{i \in I} a_{i j}^{B}-\sum_{k \in K} b_{j k}^{B}=0 \quad \forall j \in J \\
& \sum_{j \in J} b_{j k}^{P}=d_{k} \quad \forall k \in K
\end{aligned}
$$




$$
\begin{aligned}
& \sum_{j \in J} b_{j k}^{B}=r_{d} d_{k},\left(0<r_{d} \leq 1\right) \quad \forall k \in K \\
& x_{j}^{R} \in\{0,1\} \quad \forall j \in J \\
& x_{j}^{U} \in\{0,1\} \quad \forall j \in J \\
& a_{i j}^{P} \geq 0 \quad \forall i \in I, \forall j \in J \\
& a_{i j}^{P} \geq 0 \quad \forall i \in I, \forall j \in J \\
& b_{j k}^{P} \geq 0 \quad \forall j \in J, \forall k \in K \\
& b_{j k}^{B} \geq 0 \quad \forall j \in J, \forall k \in K
\end{aligned}
$$

Eq. (5) states the objective function minimizes the total fixed cost for RRS and URS as well as the shipping cost, handling cost and transportation cost for primary and backup assignments. Primary assignment assumes with probability $1-q$, and backup assignment considered with probability $q$. Eq. (6) states that either of RRS or URS can be open, but not both. Eq. (7) requires opening at least one RRS. Eqs. (8) and (9) are capacity constraint for RS as primary and backup assignment, respectively. Eqs. (10) and (11) are upper bounds for the available supply as primary and backup assignment, respectively. Eqs. (12) and (13) are lower bounds for the available supply as primary and backup assignment, respectively. Eqs. (14) and (15) are balances of product flow as primary and backup assignment, respectively. Eqs. (16) and (17) mean demand of every customer must be satisfied as primary and backup assignment, respectively. Eqs. (18) and (19) are integrality restrictions on decision variables. Eqs. (20)-(23) are nonnegative constraints for primary and backup assignment amounts.

\subsection{Multi-Single Allocation Model}

The model for MSA is described as follows:

$$
\begin{aligned}
& \text { Minimize } \sum_{j \in J} F_{j}^{U} x_{j}^{U}+\sum_{j \in J} F_{j}^{R} x_{j}^{R} \\
& +(1-q)\left(\sum_{i \in I} \sum_{j \in J}\left(C_{i}^{P}+T 1_{i j}^{P}\right) a_{i j}^{P}+\sum_{j \in J} \sum_{k \in K}\left(H_{j}^{P}+T 2_{j k}^{P}\right) d_{k} y_{j k}^{P}\right) \\
& +(q)\left(\sum_{i \in I} \sum_{j \in J}\left(C_{i}^{B}+T 1_{i j}^{B}\right) a_{i j}^{B}+\sum_{j \in J} \sum_{k \in K}\left(H_{j}^{B}+T 2_{j k}^{B}\right) d_{k} y_{j k}^{B}\right)
\end{aligned}
$$

Subject to:

$$
\begin{aligned}
& \text { Eqs. (6)-(13) and (18)-(21) } \\
& \sum_{j \in J} y_{j k}^{P}=1 \quad \forall k \in K \\
& \sum_{j \in J} y_{j k}^{B}=1 \quad \forall k \in K \\
& y_{j k}^{P} \leq x_{j}^{U}+x_{j}^{R} \quad \forall j \in J, \forall k \in K \\
& y_{j k}^{B} \leq x_{j}^{R} \quad \forall j \in J, \forall k \in K \\
& \sum_{i \in I} a_{i j}^{P}-\sum_{k \in K} d_{k} y_{j k}^{P}=0 \quad \forall j \in J \\
& \sum_{i \in I} a_{i j}^{B}-\sum_{k \in K} r_{d} d_{k} y_{j k}^{B}=0 \quad \forall j \in J \\
& y_{j k}^{P} \in\{0,1\} \quad \forall j \in J, \forall k \in K
\end{aligned}
$$

$$
y_{j k}^{B} \in\{0,1\} \quad \forall j \in J, \forall k \in K
$$

In MSA model, explanation of the objective function and constraints are all equal to the MMA model except for Eqs. (25), (26), (31) and (32). These equations express that each customer must be assigned to single RS both for the primary and backup assignment, respectively. Eqs. (27)-(30) correspond to Eqs. (14)-(17) with each other.

\subsection{Single-Single Allocation Model}

The model for SSA is described as follows:

$$
\begin{aligned}
& \text { Minimize } \sum_{j \in J} F_{j}^{U} x_{j}^{U}+\sum_{j \in J} F_{j}^{R} x_{j}^{R} \\
&+(1-q)\left(\sum_{i \in I} \sum_{j \in J}\left(C_{i}^{P}+T 1_{i j}^{P}\right) a_{i j}^{P} z_{i j}^{P}+\sum_{j \in J} \sum_{k \in K}\left(H_{j}^{P}+T 2_{j k}^{P}\right) d_{k} y_{j k}^{P}\right) \\
&+(q)\left(\sum_{i \in I} \sum_{j \in J}\left(C_{i}^{B}+T 1_{i j}^{B}\right) a_{i j}^{B} z_{i j}^{B}+\sum_{j \in J} \sum_{k \in K}\left(H_{j}^{B}+T 2_{j k}^{B}\right) d_{k} y_{j k}^{B}\right)
\end{aligned}
$$

Subject to:

Eqs. (6), (7), (18)-(25), (25)-(28), (31), and (32)

$$
\begin{aligned}
& \sum_{i \in I} z_{i j}^{P}=1 \quad \forall j \in J \\
& \sum_{i \in I} z_{i j}^{B}=1 \quad \forall j \in J \\
& \sum_{i \in I} a_{i j}^{P} z_{i j}^{P} \leq U_{j}\left(x_{j}^{U}+x_{j}^{R}\right) \quad \forall j \in J \\
& \sum_{i \in I} a_{i j}^{B} z_{i j}^{B} \leq U_{j}\left(r^{R} x_{j}^{R}+r^{U} x_{j}^{U}\right) \quad \forall j \in J \\
& \sum_{j \in J} a_{i j}^{P} z_{i j}^{P} \leq P U_{i} \quad \forall i \in I \\
& \sum_{j \in J} a_{i j}^{B} z_{i j}^{B} \leq r_{p} P U_{i},\left(0<r_{p} \leq 1\right) \quad \forall i \in I \\
& \sum_{j \in J} a_{i j}^{P} z_{i j}^{P} \geq P L_{i} \quad \forall i \in I \\
& \sum_{j \in J} a_{i j}^{B} z_{i j}^{B} \geq P L_{i} \quad \forall i \in I \\
& \sum_{i \in I} a_{i j}^{P} z_{i j}^{P}-\sum_{k \in K} d_{k} y_{j k}^{P}=0 \quad \forall j \in J \\
& \sum_{i \in I} a_{i j}^{B} z_{i j}^{B}-\sum_{k \in K} r_{d} d_{k} y_{j k}^{B}=0 \quad \forall j \in J \\
& z_{i j}^{P} \in\{0,1\} \quad \forall i \in I, \forall j \in J \\
& z_{i j}^{B} \in\{0,1\} \quad \forall i \in I, \forall j \in J
\end{aligned}
$$

Eqs. (34), (35), (44), and (45) are necessary to be a single allocation for DC. Eqs. (36)-(43) correspond to Eqs. (8)-(15) with each other. Since this model involves bi-linear terms like $a^{P}{ }_{i j} z_{i j}$ and $a^{B}{ }_{i j} z^{B}{ }_{i j}$, we need to introduce new variables and the additional constraints to linearize them as follows:

$$
\begin{aligned}
Z_{i j}^{P} & =a_{i j}^{P} z_{i j}^{P} \\
Z_{i j}^{B} & =a_{i j}^{B} z_{i j}^{B}
\end{aligned}
$$


$a_{i j}^{P}-Z_{i j}^{P} \geq 0 \quad \forall i \in I, \forall j \in J$

$a_{i j}^{B}-Z_{i j}^{B} \geq 0 \quad \forall i \in I, \forall j \in J$

$a_{i j}^{P}-B \leq Z_{i j}^{P}-B z_{i j}^{P} \quad \forall i \in I, \forall j \in J$

$a_{i j}^{B}-B \leq Z_{i j}^{B}-B z_{i j}^{B} \quad \forall i \in I, \forall j \in J$

where $B$ is a certain large value.

\section{NUMERICAL EXPERIMENT}

In this section, we perform numerical experiments to evaluate the $w$-model performance and compare it to the $w / o$-model of every system parameters. The problem instances were randomly generated. We set the fixed charges for opening RS, operational costs and customer demands are the same for both models. At first, small and large size problems are considered. The numerical experiments are performed for three-allocation models, MMA, MSA and SSA. For small size problem, the data used to run the numerical experiments are summarized in Tables 1 and 2. The numerical experiments are solved by ILOG CPLEX 12.2 optimization software using a computer with $2.66 \mathrm{GHz}$ core 2 duo processor and $2 \mathrm{~GB}$ of RAM.

Every node denoting the members of the facilities is generated randomly. The distance between them is calculated on a basis of Euclidian norm. Then, we get the unit transportation cost by multiplying the unit 1.5 and 1.0 with the distance between DC to RS and RS to customer, respectively.

Table 1. Parameter values for small size model

\begin{tabular}{cccccc}
\hline $\begin{array}{c}\text { Para- } \\
\text { meter }\end{array}$ & $\mathrm{RS}_{1}$ & $\mathrm{RS}_{2}$ & $\mathrm{RS}_{3}$ & $\mathrm{RS}_{4}$ & $\mathrm{RS}_{5}$ \\
\hline$F_{j}^{U}$ & 59720 & 98720 & 71720 & 20720 & 41720 \\
$F_{j}^{R}$ & 111600 & 150600 & 123600 & 72600 & 93600 \\
$H_{j}^{P}$ & 60 & 82 & 60 & 74 & 86 \\
$H_{j}^{B}$ & 116 & 127 & 126 & 128 & 136 \\
$U_{j}$ & 2740 & 6210 & 3030 & 750 & 1470 \\
$T 1_{l j}^{P}$ & 1500 & 1125 & 1350 & 1080 & 1020 \\
$T 1^{P}{ }_{2 j}$ & 615 & 150 & 420 & 120 & 420 \\
\hline
\end{tabular}

Table 2. Parameter values

\begin{tabular}{ccccc}
\hline \multirow{2}{*}{$\mathrm{DC}$} & \multicolumn{2}{c}{$r_{p}=0.8$} & \multicolumn{2}{c}{$r_{p}=1.0$} \\
\cline { 2 - 5 } & $\mathrm{DC}_{1}$ & $\mathrm{DC}_{2}$ & $\mathrm{DC}_{1}$ & $\mathrm{DC}_{2}$ \\
\hline$P U_{i}$ & 5050 & 3680 & 5050 & 3680 \\
$P L_{i}$ & 500 & 290 & 500 & 290 \\
$C_{i}^{P}$ & 77 & 98 & 77 & 98 \\
$C_{i}^{B}$ & 93 & 108 & 77 & 98 \\
\hline
\end{tabular}

\subsection{Results for Small Sized Model}

The scale of the numerical experiment is shown as follows: the number of DCs is 2, the number of RSs is 5 , and the number of REs is 50 (hereinafter such a feature will be denoted as (2-5-50)). In Table 3, we show the comparison of the problem sizes among MMA, MSA and SSA models. MMA and MSA are a MIP problem while SSA is just an integer programming problem. The parameters of continuity rate for these numerical experiments are given as follows: $r^{U}=0.2, r^{R}=0.8, r_{p}=0.8$, $r_{h}=0.5$. For simplicity, we suppose an identical disruption probability $(q)$ for all RSs and vary $q$ from $0.1-0.5$ for abnormal situation.

Table 3. Number of decision variables and constraints

\begin{tabular}{cccc}
\hline Model & $\begin{array}{c}\text { Real variable } \\
\text { number }\end{array}$ & $\begin{array}{c}0-1 \text { variable } \\
\text { number }\end{array}$ & $\begin{array}{c}\text { Constraint } \\
\text { number }\end{array}$ \\
\hline MMA & 520 & 10 & 134 \\
MSA & 520 & 510 & 634 \\
SSA $^{*}$ & 520 & 530 & 856 \\
\hline
\end{tabular}

${ }^{*}$ After linearization.

We evaluate how the change in the critical parameters such as the disruption probability in the RS affects the relative difference $(\mathrm{RD})$ between the expected costs of $w / o$ and $w$-model. RD is defined by the Eq. (52).

$$
R D(\%)=\frac{\text { EC of } w / o \text { model }- \text { EC of } w \text { model }}{\text { EC of } w \text { model }}
$$

where EC stands for expected cost.

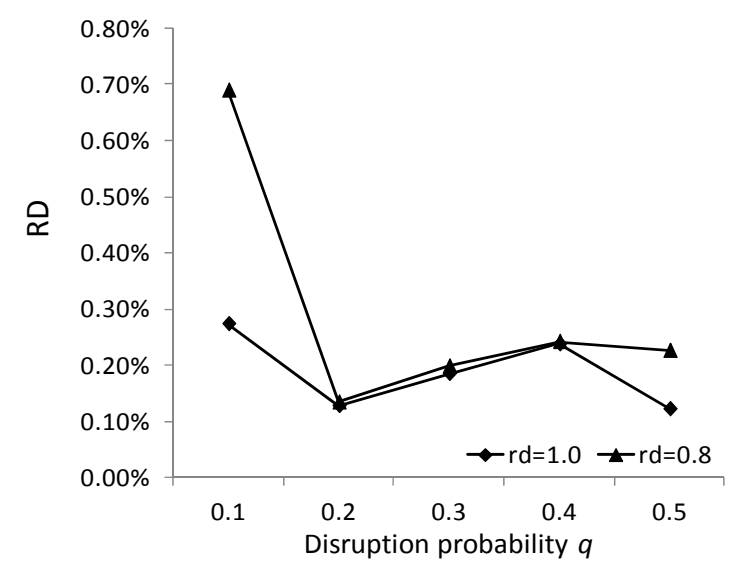

Figure 7. Relative difference (RD) against disruption probability $(q)$.

In Figure 7, we visualize RD when $r_{d}=0.8$ and $r_{d}=$ 1.0 for MMA allocation model. The cost is fluctuated and tends to decline as $q$ grows. Since the $w$-model is more flexible than the $w / o$-model as generic nature, the $w$-model outperforms the $w / o$-model for all disruption 
Table 4. Comparison result among three models for (2-5-50) problem $\left(r_{d}=0.8\right)$

\begin{tabular}{|c|c|c|c|c|c|c|c|c|c|c|c|c|c|}
\hline \multirow{3}{*}{\multicolumn{2}{|c|}{$\begin{array}{c}\text { Probability }(q) \\
\text { Model type }\end{array}$}} & \multicolumn{4}{|c|}{0.1} & \multicolumn{4}{|c|}{0.3} & \multicolumn{4}{|c|}{0.5} \\
\hline & & \multicolumn{2}{|c|}{$w$} & \multicolumn{2}{|c|}{$w / o$} & \multicolumn{2}{|c|}{$w$} & \multicolumn{2}{|c|}{$w / o$} & \multicolumn{2}{|c|}{$w$} & \multicolumn{2}{|c|}{$w / o$} \\
\hline & & URS & RRS & URS & RRS & URS & RRS & URS & RRS & URS & RRS & URS & RRS \\
\hline \multirow{3}{*}{$\begin{array}{l}\text { No. of facili- } \\
\text { ties (RS\#) }\end{array}$} & MMA & $2(\# 1,4)$ & $1(\# 2)$ & $1(\# 4)$ & $2(\# 1,2)$ & $1(\# 4)$ & $2(\# 1,2)$ & $1(\# 4)$ & $2(\# 1,2)$ & $2(\# 1,5)$ & $2(\# 2,4)$ & $1(\# 4)$ & $2(\# 1,2)$ \\
\hline & MSA & $1(\# 4)$ & $2(\# 1,2)$ & $1(\# 4)$ & $2(\# 1,2)$ & $1(\# 4)$ & $2(\# 1,2)$ & $1(\# 4)$ & $2(\# 1,2)$ & 0 & $3(\# 1,2,4)$ & $1(\# 4)$ & $2(\# 1,2)$ \\
\hline & SSA & 0 & $2(\# 1,2)$ & 0 & $2(\# 1,2)$ & 0 & $2(\# 1,2)$ & 0 & $2(\# 1,2)$ & 0 & $2(\# 1,2)$ & 0 & $2(\# 1,2)$ \\
\hline \multirow{3}{*}{$\begin{array}{c}\text { Fixed } \\
\text { cost }\end{array}$} & MMA & 80440 & 150600 & 20720 & 262200 & 20720 & 262200 & 20720 & 262200 & 101440 & 223200 & 20720 & 244200 \\
\hline & MSA & 20720 & 262200 & 20720 & 262200 & 20720 & 262200 & 20720 & 262200 & 0 & 334800 & 20720 & 262200 \\
\hline & SSA & 0 & 262200 & 0 & 262200 & 0 & 262200 & 0 & 262200 & 0 & 262200 & 0 & 262200 \\
\hline \multirow{3}{*}{$\begin{array}{c}\text { Normal } \\
\text { cost }\end{array}$} & MMA & \multicolumn{2}{|c|}{$3,342,900$} & \multicolumn{2}{|c|}{$3,342,900$} & \multicolumn{2}{|c|}{$3,342,900$} & \multicolumn{2}{|c|}{$3,342,900$} & \multicolumn{2}{|c|}{$3,338,680$} & \multicolumn{2}{|c|}{$3,372,700$} \\
\hline & MSA & \multicolumn{2}{|c|}{$3,343,350$} & \multicolumn{2}{|c|}{$3,343,350$} & \multicolumn{2}{|c|}{$3,343,350$} & \multicolumn{2}{|c|}{$3,343,350$} & \multicolumn{2}{|c|}{$3,343,350$} & \multicolumn{2}{|c|}{$3,343,350$} \\
\hline & SSA & \multicolumn{2}{|c|}{$3,418,550$} & \multicolumn{2}{|c|}{$3,418,550$} & \multicolumn{2}{|c|}{$3,418,550$} & \multicolumn{2}{|c|}{$3,418,550$} & 3,41 & 8,550 & 3,41 & 8,550 \\
\hline Abnormal & MMA & 4,268 & 652 & 4,00 & 2,700 & 3,97 & 7,380 & 4,00 & 2,700 & 3,89 & 7,900 & 4,00 & 1,200 \\
\hline cost & MSA & 4,511 & ,957 & 4,51 & 2,182 & 4,51 & 2,182 & 4,51 & ,957 & 4,39 & 5,277 & 4,51 & 1,957 \\
\hline & SSA & 4,511 & ,957 & 4,51 & 1,957 & 4,51 & 1,957 & 4,51 & ,957 & 4,51 & 1,957 & 4,51 & 1,957 \\
\hline Expected & MMA & 3,666 &, 515 & 3,69 & 1,800 & 3,81 & 6,164 & 3,82 &, 760 & 3,94 & 2,930 & 3,95 & 1,870 \\
\hline cost & MSA & 3,743 & 153 & 3,74 & 3,131 & 3,97 & 6,852 & 3,97 & 5,944 & 4,21 & 0,574 & 4,20 & 4,114 \\
\hline & SSA & 3,790 &, 091 & 3,79 & 0,091 & 4,00 & 8,772 & 4,00 & 3,772 & 4,22 & 7,454 & 4,22 & 27,454 \\
\hline CPU time & MMA & 0.0 & & & 06 & & 05 & & 09 & & .08 & & .08 \\
\hline [s] & MSA & 0.5 & & & 55 & & 53 & & 47 & & .02 & & .78 \\
\hline & SSA & 0.0 & 88 & & 05 & & 05 & & 05 & & .06 & & .05 \\
\hline GAP (\%) & MMA & 0.0 & 00 & & 00 & & 00 & & 00 & & .52 & & .00 \\
\hline & MSA & 0.2 & 24 & & 02 & & 02 & & 99 & & .02 & & .25 \\
\hline & SSA & 0.0 & 33 & & 03 & & 01 & & 01 & & .01 & & .01 \\
\hline RD (\%) & MMA & & 0. & & & & & & & & 0.2 & & \\
\hline & MSA & & 0. & & & & & & & & -0.1 & & \\
\hline & SSA & & 0. & & & & & & & & 0.0 & & \\
\hline
\end{tabular}

URS: unreliable relay station, RRS: reliable relay station, RS: relay station, RD: relative difference.

probability. The decrease of RD implies that once the network is disrupted, the model intensifies to open more reliable RSs rather than unreliable ones. This leads the increase of operational cost because abnormal cost becomes higher.

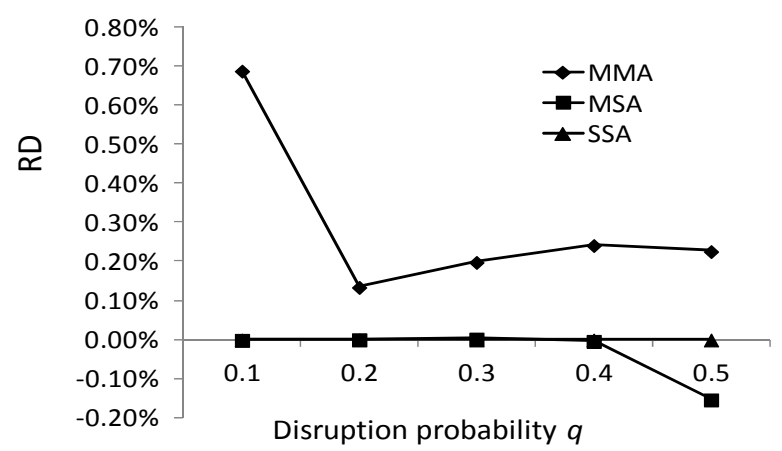

Figure 8. Relative difference (RD) of expected cost comparison between multi-multi allocation model (MMA), multi-single allocation model (MSA), and single-single allocation model (SSA). $r^{U}=$ $0.2, r^{R}=0.8, \mathrm{r}_{p}=0.8, r_{h}=0.5, r_{\mathrm{d}}=0.8$.
In the case of $r_{d}=0.8$, the $w$-model outperformed the $w / o$-model for all disruption probability. We obtained the highest RD when disruption probability $(q)$ is at 0.1 . This condition is achieved because the $w$-model opens more unreliable facility than the $w / o$-model. In the case of $q=0.5$, the $w$-model needs to open another URS to handle backup assignment in the abnormal situation. By opening another URS, the total fixed charge becomes higher compared to the w/o-model, but it can reduce the operational cost.

In Figure 8, we show the experimental result for each allocation model MMA, MSA and SSA for disruption probability $q=0.1-0.5$. In these experiments, RD values of MSA and SSA models are almost $0.00 \%$ for all disruption probability. This is because numbers of open facilities are the same between the $w / o$ - and $w$ model for each disruption probability. The operational costs for normal and abnormal situation are also the same for both models. This condition causes the expected cost for two models to be almost the same. We have summarized the comparison result among three models for small size problem in Table 4.

The main contribution of cost deduction in the $w$ - 
model is the ability of URSs to allocate some of backup assignment to customers. This decision will reduce the abnormal cost and will influence the reduction of the expected cost in general. This decision tries to activate the function of the URS, which is cheaper than RRS, as the backup assignment in an abnormal situation. We also note that the capacity of RS is also crucial in this model. When the RS is opened as unreliable one with higher capacity, the backup ability becomes also higher in the abnormal situation.

Figure 9 illustrates the different allocation model configurations between the $w / o$ - and $w$-model for disrupttion probability $q=0.3$. This figure describes the robustness and flexibility of the $w$-model in an abnormal situation. On the other hand, the w/o-model becomes more rigid in an abnormal situation since URS completely stops the operation and RRS will provide all customers' demand.

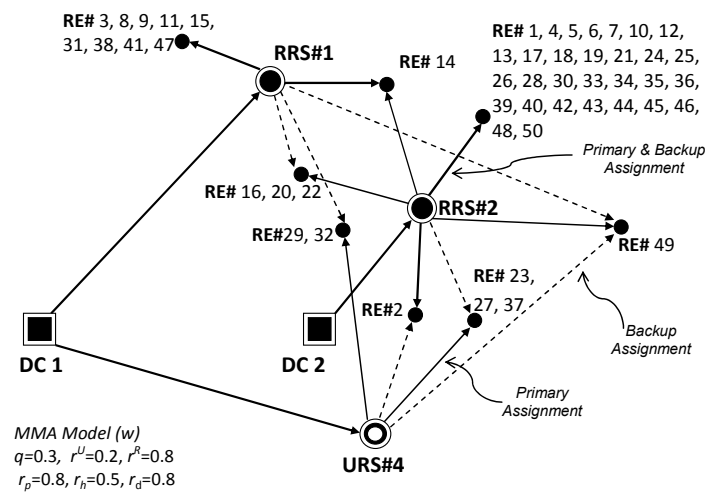

(a)

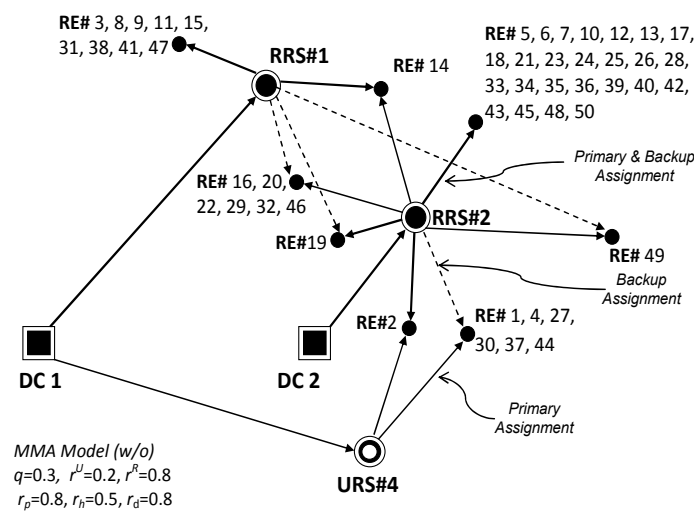

(b)

Figure 9. Allocation model comparison for multi-multi allocation model (MMA) between the $w$-model (a) and w/o-model (b) with $q=0.3, r_{p}=0.8, r_{d}=0.8$. DC: distribution center, RE: customer, RRS: reliable relay station, URS: unreliable relay station.

\subsection{Results for Large Sized Model}

To investigate further validity and properties of each model, we conducted additional experiments with larger data sizes. These problems are (3-10-100), (4-15$150)$, and (6-25-250). The parameters of continuity rate for these numerical experiments are given as follows: $r^{U}$ $=0.2, r^{R}=0.8, r_{p}=0.8, r_{h}=0.5$ and $r_{d}=0.8$. In these cases, we cannot obtain the feasible solutions of SSA model due to the difficulty of solution. Just like the small size problem, URS will shift to RRS along with the increase in the disruption probability $(q)$. In addition, the computational time becomes longer as the problem size becomes larger, especially for MSA allocation model due to having a large number of binary variables. For example, we needed more than 150 seconds to solve the data size (6-25-250).

In Figure 10, we show the difference cost regarding opening and operating the facilities between the $w / o$ and $w$-model for problem (4-15-150).

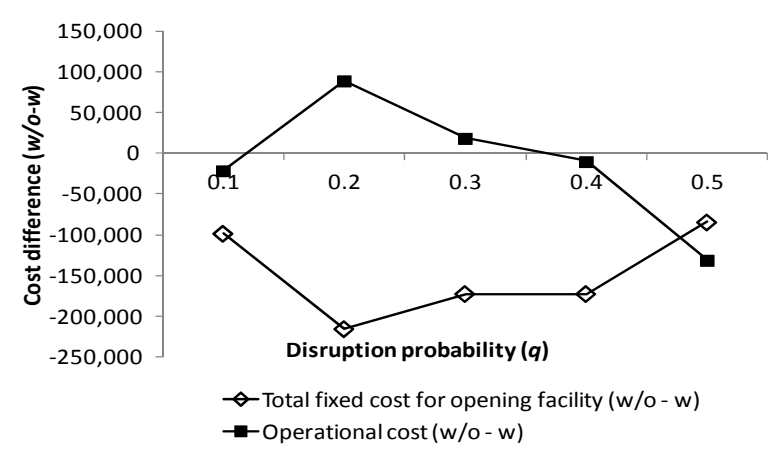

(a)

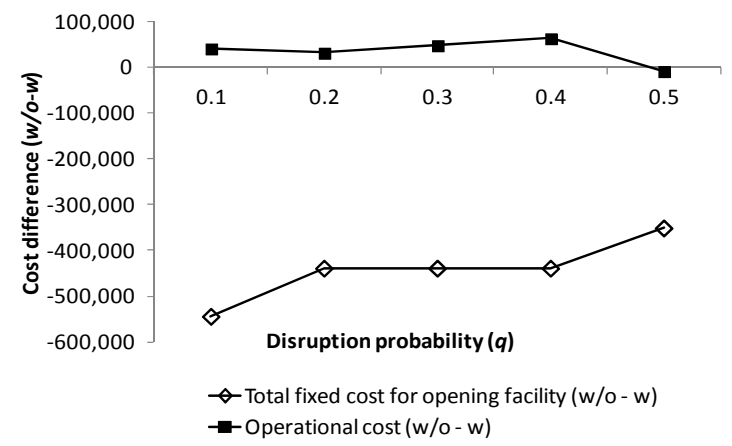

(b)

Figure 10. Cost difference between the $w / o$ - and $w$-model for multi-multi allocation model (a) and multisingle allocation model (b) allocation model with problem size (4-15-150), and parameters such like $r^{U}=0.2, r^{R}=0.8, r_{p}=0.8, r_{h}=0.5, r_{\mathrm{d}}=$ 0.8 .

For small disruption probability, the $w$-model tends to open more URS than the $w / o$-model by virtue of the backup capabilities of URS in an abnormal situation. In the certain condition, we lead to cost saving by opening unreliable facility instead of opening reliable one. Figure 11 depicts the relation between disruption probability and the number of relay stations for the $w / o$ and $w$ model.

Consequently, the result confirms that the $w$-model 
provides more flexibility than the $w / o$-model where facility is not fully halted due to disruption, but still have the ability to serve the customers depending on the continuity rate.

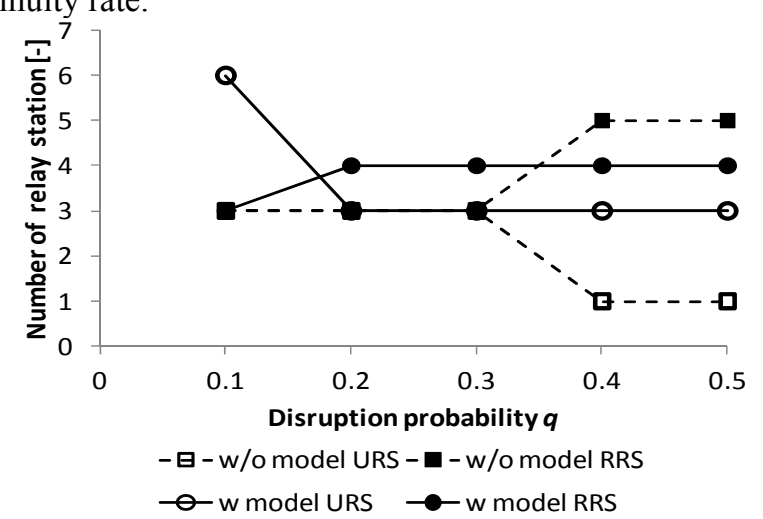

(a)

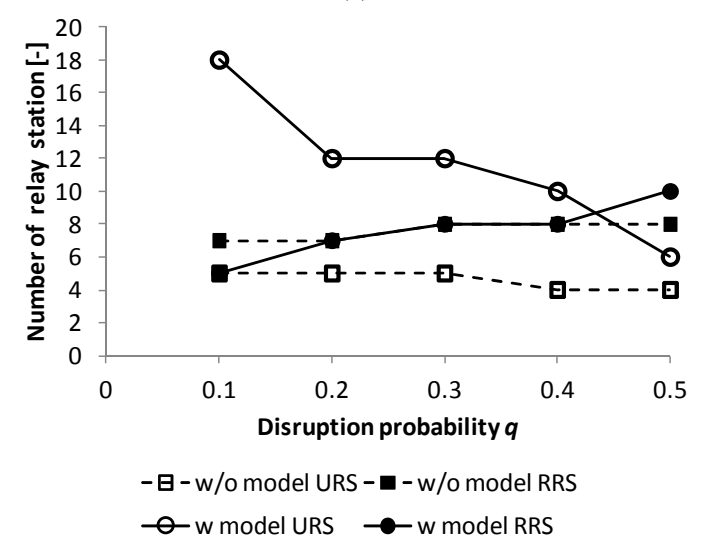

(b)

Figure 11. Profile of open RSs with disruption probability $\left(r^{U}=0.2, r^{R}=0.8, r_{d}=0.8\right)$. (a) $(3-10-100)$ problem, (b) (6-25-250) problem. RRS: reliable relay station, URS: unreliable relay station.

\section{CONCLUSION}

In this paper, we proposed a new model for supply chain network design by introducing the continuity rate. The proposed model was successful in designing RSs, which are more robust and flexible in an abnormal situation. The continuity rate is a new parameter to formulate the models more practically and make the analysis more comprehensively. Models that consider the continuity rate in practice can lead to additional costs such as handling cost and transportation cost which are usually more expensive in abnormal situations. The optimization problem is formulated as a MIP model. Through numerical experiments, we have shown that the proposed approach is promising for designing resilient logistic networks available for real-world mitigation planning.

For future works, one possible extension is to consider sharing product between RS when disruption occurs. Considering this condition in the model will reduce backup transportation cost in an abnormal situation. It is also possible to integrate the model with other decisions such as inventory management and production management.

\section{REFERENCES}

Berman, O., Krass, D., and Menezes, M. B. C. (2007), Facility reliability issues in network p-median problems: strategic centralization and co-location effects, Operations Research, 55(2), 332-350.

Chopra, S. and Sodhi, M. S. (2004), Managing risk to avoid supply-chain breakdown, MIT Sloan Management Review, 46(1), 53-61.

Cui, T., Ouyang, Y., and Shen, Z. J. M. (2010), Reliable facility location design under the risk of disruptions, Operations Research, 58(4-Part-1), 998-1011.

Kleindorfer, P. R. and Saad, G. H. (2005), Managing disruption risks in supply chain, Production and $O p$ erations Management, 14(1), 53-58.

Klibi, W., Martel, A., and Guitouni, A. (2010), The design of robust value-creating supply chain net-works: a critical review, European Journal of Operational Research, 203(2), 283-293.

Lim, M., Daskin, M. S., Bassamboo, A., and Chopra, S. (2009), A facility reliability problem: formulation, properties, and algorithm, Naval Research Logistics, 57(1), 58-70.

Melo, M. T., Nickel, S., and Saldanhada-Gama, F. (2009), Facility location and supply chain management: a review, European Journal of Operational Research, 196(2), 401-412.

Peng, P., Snyder, L. V., Lim, A., and Liu, Z. (2011), Reliable logistics networks design with facility disruptions, Transportation Research Part B, 45(8), 1190-1211.

Rusman, M. and Shimizu, Y. (2011), Comparison of multistage logistic network design as critical infrastructure under disruption risk, Proceedings of the 54th Japan Joint Automatic Control Conference, Aichi, Japan, 1563-1568.

Rusman, M. and Shimizu, Y. (2012), Morphological analysis for multistage logistic network optimization under disruption risk, Journal of Japan Industrial Management Association, 63(4), 289-297.

Schmitt, A. J. (2011), Strategies for customer service level protection under multi-echelon supply chain disruption risk, Transportation Research Part B, 45(8), 1266-1283.

Shimizu, Y., Yamazaki, Y., and Wada, T. (2006), A flexible design for logistic network under uncertain demands through hybrid meta-heuristic strategy, Transactions of the Institute of Systems, Control and In- 
formation Engineers, 19(9), 342-349.

Shimizu, Y., Yamazaki, Y., and Wada, T. (2008), Multimodal logistics network design over planning horizon through a hybrid meta-heuristic approach, Journal of Advanced Mechanical Design, Systems, and Manufacturing, 2(5), 915-925.

Shimizu, Y., Fushimi, H., and Wada, T. (2011), Robust logistics network modeling and design against uncertainties, Journal of Advanced Mechanical Design, Systems, and Manufacturing, 5(2), 103-114.

Shimizu, Y. and Rusman, M. (2012), A hybrid approach for huge multi-stage logistic network optimization under disruption risk, Journal of Chemical Engineering of Japan, 45(8), 597-603.

Snyder, L.V. and Daskin, M. S. (2005), Reliability mod- els for facility location: the expected failure cost case, Transportation Science, 39(3), 400-416.

Tang, C. S. (2006), Perspectives in supply chain risk management, International Journal of Production Economics, 103(2), 451-488.

Tang, O. and Nurmaya Musa, S. (2011), Identifying risk issues and research advancements in supply chain risk management, International Journal of Production Economics, 133(1), 25-34.

Tomlin, B. (2006), On the value of mitigation and contingency strategies for managing supply chain disruption risks, Management Science, 52(5), 639-657.

Yu, H., Zeng, A. Z., and Zao, L. (2009), Single or dual sourcing: decision-making in the presence of supply chain disruption risks, Omega, 37(4), 788-800. 